\title{
Prospecção científica e tecnológica de potenciais imunoterapias incluindo vacinas anticâncer com enfoque em osteossarcoma
}

\author{
Scientific and technological prospection of potential immunotherapies including anti-cancer \\ vaccines with a focus on osteosarcoma \\ Prospección científica y tecnológica de posibles inmunoterapias, incluidas las vacunas contra el \\ cáncer, con especial atención al osteosarcoma
}

Tatiane Batista dos Santos

ORCID: https://orcid.org/0000-0002-5807-4614 Universidade Tiradentes, Brasil

E-mail: Tatiane.bdos@ souunit.com.br

Denilson dos Santos Gomes

ORCID: https://orcid.org/0000-0003-4462-693X Universidade Tiradentes, Brasil

E-mail: Denilsongomes123@icloud.com

Alícia Beatriz Fontes de Sousa

ORCID: https://orcid.org/0000-0002-3718-5876 Universidade Tiradentes, Brasil

E-mail: alicia.fontes@ souunit.com.br

Ítalo Samuel Gonçalves Rodrigues

ORCID: https://orcid.org/0000-0002-7053-2235 Universidade Tiradentes, Brasil

E-mail: italosanmuel@ hotmail.com

Francine Ferreira Padilha

ORCID: https://orcid.org/0000-0002-5892-4252 Universidade Tiradentes, Brasil E-mail: fpadilha@yahoo.com

Daniela Droppa-Almeida

ORCID: https://orcid.org/0000-0002-8154-1030 Universidade Tiradentes, Brasil

E-mail: danieladroppa@gmail.com

\begin{abstract}
Resumo
Osteossarcoma (OS) é um tumor ósseo maligno, cujo sua terapêutica é realizada através da remoção cirúrgica associado a quimioterapia combinada, a qual incluem a utilização de medicamentos. Com intuito de verificar a importância da imunoterapia no cunho científico, foram realizadas pesquisas a fim de se confirmar a relevância que o estudo apresenta como inovação tecnológica. Para isso as bibliotecas de artigos do PubMed e Science Direct foram acessadas para a realização de uma prospecção científica, um quantitativo de 278 e $40 \mathrm{com}$ as associações dos descritores "Bone Neoplasms AND cancer vaccines" e "Osteosarcoma AND cancer vaccines" foram obtidos, respectivamente. Em relação a prospecção tecnológica utilizou-se os mesmos descritores e as buscas foram realizadas no Google Patents selecionando os escritórios WO e BR quando necessário, com o uso dos descritores "bone neoplasms", "osteosarcoma" e "cancer vaccines", apresentando um total de 62.610, 20410 e 34337 patentes, respectivamente para o escritório WO. Já no escritório BR foram encontrados 687, 613 e 585 respectivamente. Dentre esses achados foi possível perceber que a busca por imunoterapias para OS é crescente, no entanto, o uso de vacinas para OS foi mais presente na prospecção científica do que na tecnológica, isso pode ser atribuído a tecnologia das vacinas terapêuticas serem recentes. A partir desses resultados pode-se concluir que o estudo forneceu dados de prospecção científico-tecnológico, que pode auxiliar no direcionamento de pesquisas, ressaltando a relevância da análise prospectiva tecnológica, como oportunidade de investir na propriedade intelectual como solução das necessidades sociais e econômicas.
\end{abstract}

Palavras-chave: Imunoterapia; Osteossarcoma; Vacinas anticâncer.

\section{Abstract}

Osteosarcoma (OS) is a malignant bone tumor whose treatment is performed through surgical removal associated with combined chemotherapy, which include the use of medications. In order to verify the importance of immunotherapy in a scientific way, researches were carried out in order to confirm the relevance that the study presents as a technological 
innovation. For this, the PubMed and Science Direct article libraries were accessed to carry out a scientific prospection, a quantity of 278 and 40 with the associations of the descriptors "Bone Neoplasms AND cancer vaccines" and "Osteosarcoma AND cancer vaccines" were obtained, respectively. Regarding technological prospecting, the same descriptors were used and searches were performed in Google Patents, selecting the WO and BR offices when necessary, using the descriptors "bone neoplasms", "osteosarcoma" and "cancer vaccines", presenting a a total of 62,610, 20410 and 34337 patents, respectively for the WO office. In the BR office, 687, 613 and 585 respectively were found. Among these findings, it was possible to notice that the search for immunotherapies for OS is growing, however, the use of vaccines for OS were more present in scientific than in technological prospecting, this can be attributed to the technology of therapeutic vaccines being recent. From these results, it can be concluded that the study provided data from scientifictechnological prospection, which can help direct research, highlighting the relevance of prospective technological analysis as an opportunity to invest in intellectual property as a solution to social and economic needs.

Keywords: Immunotherapy; Osteosarcoma; Anticancer vaccines.

\section{Resumen}

El osteosarcoma (OS) es un tumor óseo maligno cuyo tratamiento se realiza mediante la extirpación quirúrgica asociada a la quimioterapia combinada, que incluye el uso de medicamentos. Con el fin de verificar la importancia de la inmunoterapia de manera científica, se realizaron investigaciones con el fin de confirmar la relevancia que presenta el estudio como innovación tecnológica. Para ello, se accedió a las bibliotecas de artículos PubMed y Science Direct para realizar una prospección científica, se obtuvo una cantidad de 278 y 40 con las asociaciones de los descriptores "Neoplasias Óseas Y vacunas contra el cáncer" y "Osteosarcoma Y vacunas contra el cáncer", respectivamente. En cuanto a la prospección tecnológica, se utilizaron los mismos descriptores y se realizaron búsquedas en Google Patents, seleccionando las oficinas de WO y BR cuando fue necesario, utilizando los descriptores "neoplasias óseas", "osteosarcoma" y "vacunas contra el cáncer", presentando un total de 62.610, 20410 y 34337 patentes, respectivamente para la oficina WO. En la oficina BR, se encontraron 687, 613 y 585 respectivamente. Entre estos hallazgos, se pudo notar que la búsqueda de inmunoterapias para OS está creciendo, sin embargo, el uso de vacunas para la SG estuvieron más presentes en la prospección científica que en la tecnológica, esto se puede atribuir a que la tecnología de las vacunas terapéuticas es reciente. De estos resultados se puede concluir que el estudio aportó datos de prospección científicotecnológica, que pueden ayudar a orientar la investigación, destacando la relevancia del análisis tecnológico prospectivo como una oportunidad para invertir en propiedad intelectual como solución a necesidades sociales y económicas.

Palabras clave: Inmunoterapia; Osteosarcoma; Vacunas contra el cáncer.

\section{Introdução}

As neoplasias são consideradas patologias que têm em comum o crescimento alterado de células, que posteriormente invadem tecidos e órgãos. Dividindo-se rapidamente, estas células se mostram muito agressivas e incontroláveis, indicando a formação de tumores que podem se disseminar para diversas regiões do corpo (Pfeifer, Wick, 2018; Marcião et al., 2021). Quando essas descoordenações começam em tecidos epiteliais, como mucosas ou pele, são chamados de carcinomas, mas se a origem são os tecidos conjuntivos, como músculo, cartilagem ou osso, são denominados de sarcomas (Pfeifer \& Wick, 2018).

De acordo com Guerra et al., (2017)as neoplasias malignas serão a maior etiologia de morbimortalidade ultrapassando, principalmente as doenças cardiovasculares. Segundo a Sociedade Brasileira de Cancerologia, aproximadamente 2.700 novos casos de câncer ósseo são vistos como tumores raros. De tal forma que os tumores malignos são classificados em primários e secundários. As gêneses dos tumores primários são do próprio osso, por outro lado os secundários são originados de partes diferentes do organismo e logo migram para os ossos, devido a metástase (Mutsaers \& Walkley, 2014).

Dentre os tumores ósseos, o osteossarcoma (OS) é caracterizado como um tumor maligno primário em tecidos ósseos, com incidência mundial de 3,4 por milhão de pessoas por ano, o qual apresenta uma maior prevalência em crianças e adolescestes, mas também, é comum em idosos com mais de 60 anos (Corre et al., 2020; Misaghi et al., 2018). O OS apresentam algumas características morfologicamente alteradas, mais especificamente os osteblastos, os quais são responsáveis por produzir um osteóide anormal (Misaghi et al., 2018). Esse tumor acontece, preferencialmente, em algumas regiões metafisárias de ossos longos, como o úmero, a tíbia e fêmur. No entanto, também podem ser identificados em outros tipos de ossos, como nas vértebras, bacia e mandíbula (Corre et al., 2020; Mutsaers \& Walkley, 2014). 
Além disso, também é caracterizado como uma alteração cromossômica responsável por gerar uma cascata de modificações genéticas, e conhecido devido a sua complexidade de mecanismos envolvidos em sua origem, assim como pela variedade de fatores de risco predisponentes a ele, dos quais pôde-se citar o sexo, a idade, e assim como a herança genética, pois auxiliam para a evolução do OS (Saba et al., 2019; Sadykova et al., 2020). Vale ressaltar, também, mediante alguns estudos que vêm sendo realizados, o OS pode estar envolvido também em mutações nas células progenitoras mesenquimais presentes no estroma da medula óssea (Cortini et al., 2017).

Diante desse contexto, as opções de tratamento dependerão muito de algumas características do tumor, incluindo localização, volume e estadiamento, assim vão direcionar para o procedimento adequado. $O$ tratamento atual consiste na remoção cirúrgica do tumor primário associado a quimioterapia combinada, a qual incluem a utilização de metotrexato (MTX), cisplatina (CDDP), epirrubicina (EPI) ou ifosfamida (IFO)(Zhang et al., 2018). Mas mesmo com métodos de diagnósticos e terapêutica, infelizmente cerca de $65 \%$ dos pacientes com OS ainda não alcançam bons resultados. Por isso, novas formas mais eficazes devem ser investigadas para melhorar os resultados terapêuticos em pacientes com OS(Miwa et al., 2019).

Hoje existem diversos testes capazes de prever a resposta a fármacos e o risco de metástase. De forma paralela, em relação ao tratamento é possível evidenciar, estudos com estratégias baseadas em fármacos adjuvantes e quimioterápicos, mais recentemente, a utilização de imunoterapias (Cabrera-Andrade et al., 2020; Miwa et al., 2019; Younis et al., 2019). Com isso, diversas iniciativas vêm surgindo, em sua maioria com objetivo de melhorar os recursos terapêuticos, trazendo como consequência a produção de dados genômicos, o qual traz consigo uma variedade de ferramentas computacionais e de pesquisadores capazes de interpretar informações (Hu et al., 2019). A respeito disso, as vacinas terapêuticas têm ganhado espaço como uma alternativa de tratamento para os cânceres, e com auxílio dessas ferramentas, que também são conhecidas como bioinformática, é possível produzir vacinas potencialmente promissoras a partir da fisiopatologia do câncer, neste caso do OS (Lugowska et al., 2018; Miwa et al., 2019; Yalçın et al., 2021).

$\mathrm{Na}$ literatura cientifica disponível há evidencias que as vacinas proporcionam excelente potencial para novos estudos, com procedência na descoberta de novas alternativas de interesse terapêutico, tornando-se indispensável o estudo de vacinas com a perspectiva de tratamento contra o OS (Lugowska et al., 2018). Através disso, análises prospectivas, científicas e tecnológicas trazem relevância para a descoberta por novas terapêuticas. Com isso, mostra-se que essa análise faz com que os países tenham a oportunidade de investir na propriedade intelectual como solução para as problemáticas sociais e econômicas. Dessa forma, podendo gerar menos óbitos e gastos nos serviços de saúde. Diante desse contexto, o objetivo desse artigo foi realizar uma prospecção científica e tecnológica em busca de artigos e patentes com uso de vacinas anti-osteossarcoma como uma nova alternativa de terapêutica.

\section{Metodologia}

Esse estudo trata-se de uma pesquisa de caráter exploratório e quali-quantitativo, cujo levantamento dos dados para a prospecção científica e tecnológica aconteceu durante os meses de setembro/outubro de 2021. Em relação a prospecção científica foi adotada uma metodologia de revisão de literatura sistemática que busca nortear o mapeamento da temática a ser abordada de maneira mais coesa, possibilitando um melhor direcionamento na investigação (Chueke \& Amatucci, 2015).

Para encontrar os artigos e patentes usou-se primeiramente a plataforma de Descritores em Ciências da Saúde (DeCs ou $\mathrm{MeSH}$ ) para obtenção de descritores que estivessem de acordo com o tema proposto, neste contexto, foram selecionados: "Bone Neoplasms", "Osteosarcoma" e "Cancer vaccines "utilizadas isoladas e/ou combinadas entre si por meio do operador booleano AND, nos seguintes bancos de dados Science Direct e PubMed, analisando os últimos dez anos (2011 a 2021). No entanto, mesmo adicionando o filtro de 2011- 2021 os artigos encontrados tinham seus resumos analisados para verificar de fato a 
correlação com o tema proposto, os que não apresentavam correlação não eram selecionados, assim como foram excluídos artigos de revisão, afim de selecionar artigos originais experimentais propondo imunoterapias.

Para as buscas de patentes, foram utilizados os mesmos critérios, já mencionados anteriormente, referentes aos descritores, operador booleano e anos de publicação no banco de dados do Google Patentes e nesta plataforma para as buscas de patentes brasileiras os filtros em relação ao escritório de patentes foram selecionados, o primeiro com a opção "BR" enquanto o segundo em relação a linguagem foi selecionado "Português". Para as buscas internacionais o escritório de patentes foi selecionado a opção "WO" referente ao WIPO (Organização Mundial da Propriedade Intelectual) e a linguagem selecionada foi "Inglês".

\section{Resultados e Discussão}

Os descritores previamente selecionados foram utilizados para a prospeç̧ão científica que visa buscar artigos relacionados com o tema em questão. Os descritores foram obtidos no DeCs e segue um padrão que garante uma reprodutibilidade dos dados. Os mesmos descritores foram utilizados para a prospecção tecnológica pelo Google Patents, um banco de dados que permite buscar patentes depositadas ou concedidas em diversos escritórios e linguagem. Todos os achados das prospecções científicas e tecnológicas estão descritos na Tabela 1.

Tabela 1. Quantitativo de artigos e patentes encontradas nos respectivos bancos de dados.

\begin{tabular}{lcccc} 
& & & \multicolumn{3}{c}{ Google Patents } \\
& PubMed & Science Direct & WO & BR* \\
Bone Neoplasms & 70851 & 40665 & 62610 & 687 \\
Osteosarcoma & 18533 & 25113 & 20410 & 613 \\
Cancer Vaccines & 20905 & 49841 & 34337 & 585 \\
Bone Neoplasms AND cancer & 278 & 2073 & 18365 & 161 \\
vaccines & 40 & 1342 & 8018 & 159 \\
Osteosarcoma AND cancer vaccines & 40 & & & \\
\hline
\end{tabular}

*As buscas feitas no escritório brasileiros foram realizadas em português e não na língua inglesa. Fonte: Autores (2021).

Diante dos achados pode-se perceber uma quantidade muito maior quando se utiliza o descritor "Bone Neoplasms" isso acontece devido a existências de diversos tipos de neoplasias ósseas, como as primárias: osteossarcoma, condrossarcoma, sarcoma de Ewig, histiocitoma fibroso maligno, fibrossarcoma, tumor ósseo de células gigantes e cordoma, e ainda há neoplasias ósseas advindos de outros tecidos como câncer de mama, linfomas, câncer de pulmão, etc. Diante disso, as associações entre os descritores auxiliam a encontrar artigos que estão mais de acordo com a temática em estudo. E pelo PubMed é possível verificar um quantitativo de 278 e 40 com as associações "Bone Neoplasms AND cancer vaccines" e "Osteosarcoma AND cancer vaccines" respectivamente. Ao se analisar os 40 artigos encontrados com os descritores "Osteosarcoma AND cancer vaccines" foram encontrados 4 para osteossarcomas em cães, 4 para sarcomas Ewig, 2 para sarcomas gerais, 8 de vacinas para outros tipos de câncer e 2 vacinas utilizando vírus para outros tipos de câncer.

No entanto, pode-se perceber a presença de 7 artigos de revisão voltados a fornecer subsídio para imunoterapias (F et al., 2020; Ma et al., 2020) e vacinas terapêuticas como estratégia promissora para tratamento de osteossarcoma e um total de 8 artigos trazendo a utilização vacinas baseadas em células dendríticas ( $Y$ et al., 2020), peptídeos e antígenos tumorais de osteossarcomas carreados por vírus (Tadano et al., 2020). Vale ressaltar a presença de anticorpos monoclonais sendo utilizados para osteossarcoma, mesmo não sendo o enfoque do trabalho em questão, a presença de todos esses artigos indica a importância da imunoterapia como estratégia para o tratamento desse tipo de câncer. 
Ao se analisar os mesmos descritores "Osteosarcoma AND cancer vaccines" no Science Direct um quantitativo de 1342 artigos foi encontrado e para facilitar nossa busca foram analisadas as áreas mais direcionadas que estes artigos estavam organizados nessa biblioteca. É possível verificar as áreas na Figura 1.

Figura 1. Gráfico pizza apresentando as áreas em que os artigos encontrados com os descritores "Osteosarcoma AND cancer vacinnes" estão relacionados na biblioteca do Science Direct.

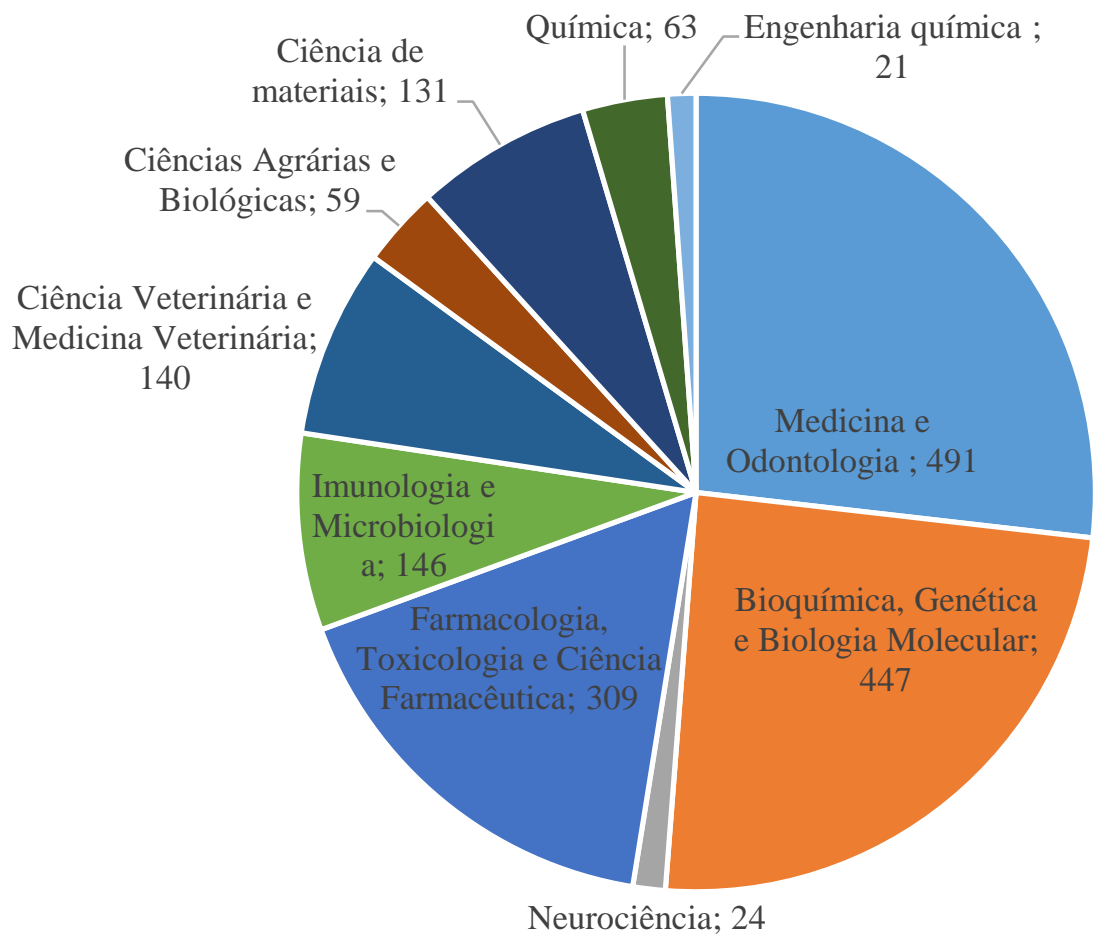

Fonte: Autores (2021).

Ao selecionar a área de "Imunologia e Microbiologia" um total de 146 artigos foram encontrados como está presente na Figura 1 e, ao se analisar esses quantitativo de artigos foram encontrados um total de 55 de revisão de literatura, 49 artigos originais, em que dentre estes a presença de artigos voltados para osteossarcoma canino e utilização de microrganismos como forma de terapia estavam bem frequentes (Martin \& Bell, 2018; Verheust et al., 2012; Watanabe et al., 2021). Mas ao se analisar os artigos voltados para os osteossarcomas em humanos pode-se encontrar vacinas de mRNA (Fan et al., 2021), vacinas com a fusão de células T $\gamma \delta$ alogênica (Watanabe et al., 2021) e células tumorais e a utilização de nano partículascarreando PD-1 (Wang et al., 2016) importante mediador de autólise celular. As vacinas terapêuticas vêm ganhando muito espaço como uma forma alternativa para o tratamento de diversos cânceres. O OS é um tumor ósseo agressivo que afeta principalmente crianças e adolescentes. OS tem uma forte tendência a recidiva e metástase, resultando em prognóstico e sobrevida ruins (Kaur Sarhadi et al., 2021). Os tratamentos atuais para OS são quimioterapia pré-operatória, ressecção cirúrgica e quimioterapia pós-operatória, que são eficazes em pacientes com OS localizada (Kaur Sarhadi et al., 2021)

No entanto, para se utilizar da imunoterapia ou qualquer outra forma de tratamento é necessário conhecer o microambiente tumoral, especialmente o microambiente ósseo, a comunicação celular e os mecanismos moleculares envolvidos na progressão tumoral (Argenziano et al., 2021). A imunoterapia possui o objetivo de ajudar o sistema imunológico a neutralizar o desenvolvimento e a progressão do câncer. O sistema imunológico normalmente protege contra ameaças externas, como infecção ou tumor, e o próprio tumor em particular limita a defesa imunológica ao liberar citocinas inibidoras ou afetando 
diretamente a expressão de marcadores de apoptose, muitas vezes. Na literatura está bem documentado o benefício derivado tanto da terapia baseada em células imunes inatas quanto no uso estimulantes imunológicos associados à quimioterapia padrão (M et al., 2021). Além disso, o OS é muito suscetível a essas estratégias terapêuticas (Mf et al., 2018), uma vez que mostra altos níveis de linfócitos CD8 + e a isso atribui-se uma alta taxa de sobrevivência (Erp et al., 2017; Ic et al., 2021), além de apresentar na superfície das células tumorais potenciais alvos para anticorpos, possibilitando uso de vacinas terapêuticas.

Ao se analisar o quantitativo de artigos por ano, foi possível perceber o crescente número de publicações com o passar dos anos. Na figura 2 é possível verificar o quantitativo de artigos encontrados no PubMed e no Science Direct distribuídos por ano com o descritor "Osteosarcoma AND cancer vaccines". Confirmando o que já foi mencionado anteriormente sobre a crescente busca de imunoterapia e vacinas anticâncer.

Figura 2. Gráfico apresentando o quantitativo de artigos encontrados no PubMed e no Science Direct com o descritor “Osteosarcoma AND cancer vaccines" distribuídos por ano, entre 2011 e 2021.

\section{Osteosarcoma AND cancer vaccines}

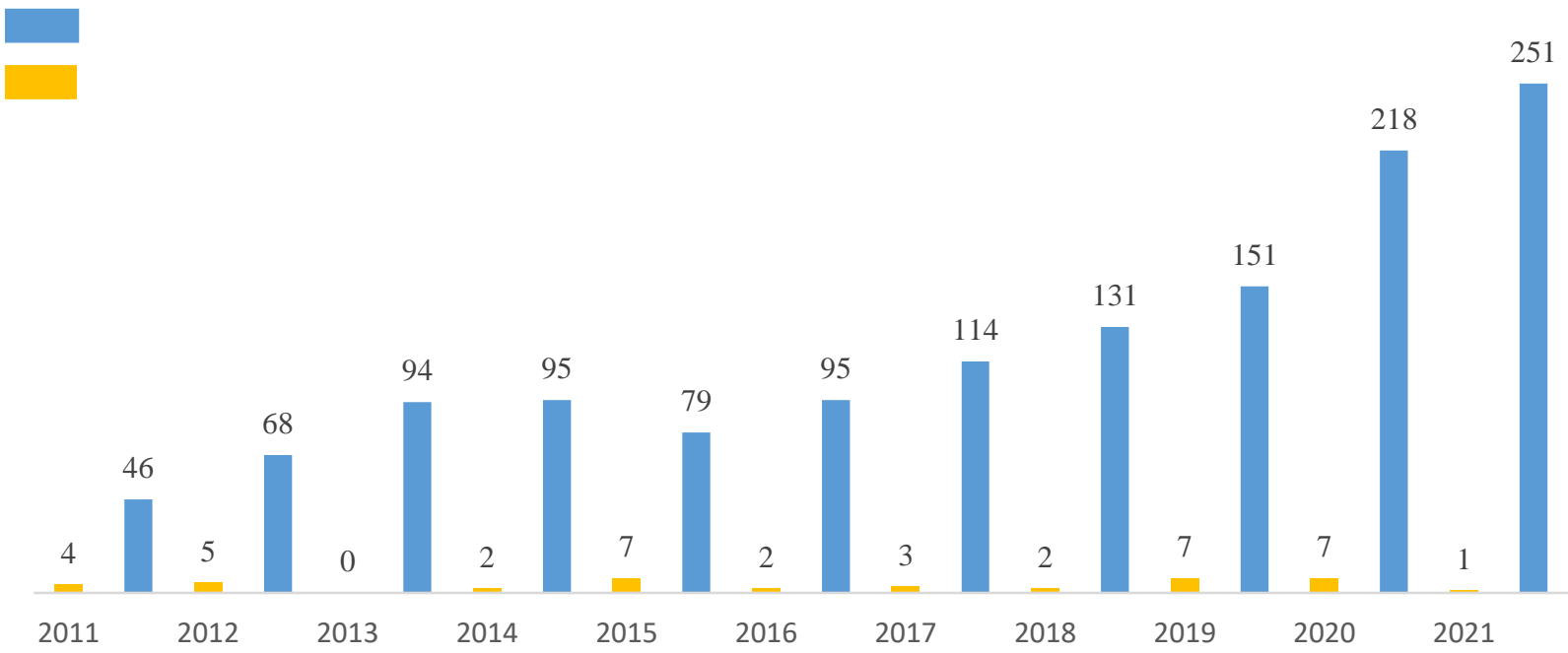

Fonte: Autores (2021).

Em relação a prospecção tecnológica como descrito na Tabela 1 pode-se notar um maior quantitativo quando se utilizou o escritório WO e isso já era esperado, visto que se trata do escritório mundial. No entanto, o mesmo pode-se perceber com os descritores utilizados, a associação entre "Osteosarcoma AND cancer vaccines" foi o que conseguiu direcionar melhor nas buscas, totalizando 8018 invenções. Na Figura 3 está presente os códigos internacionais de patentes mais relacionados com essas invenções encontradas com esse descritor, assim como com os demais. 
Figura 3. Gráfico apresentando os códigos internacionais de patentes associados as invenções e seu descritores no escritório WO - Mundial. Dados obtidos pelo Google Patentes.

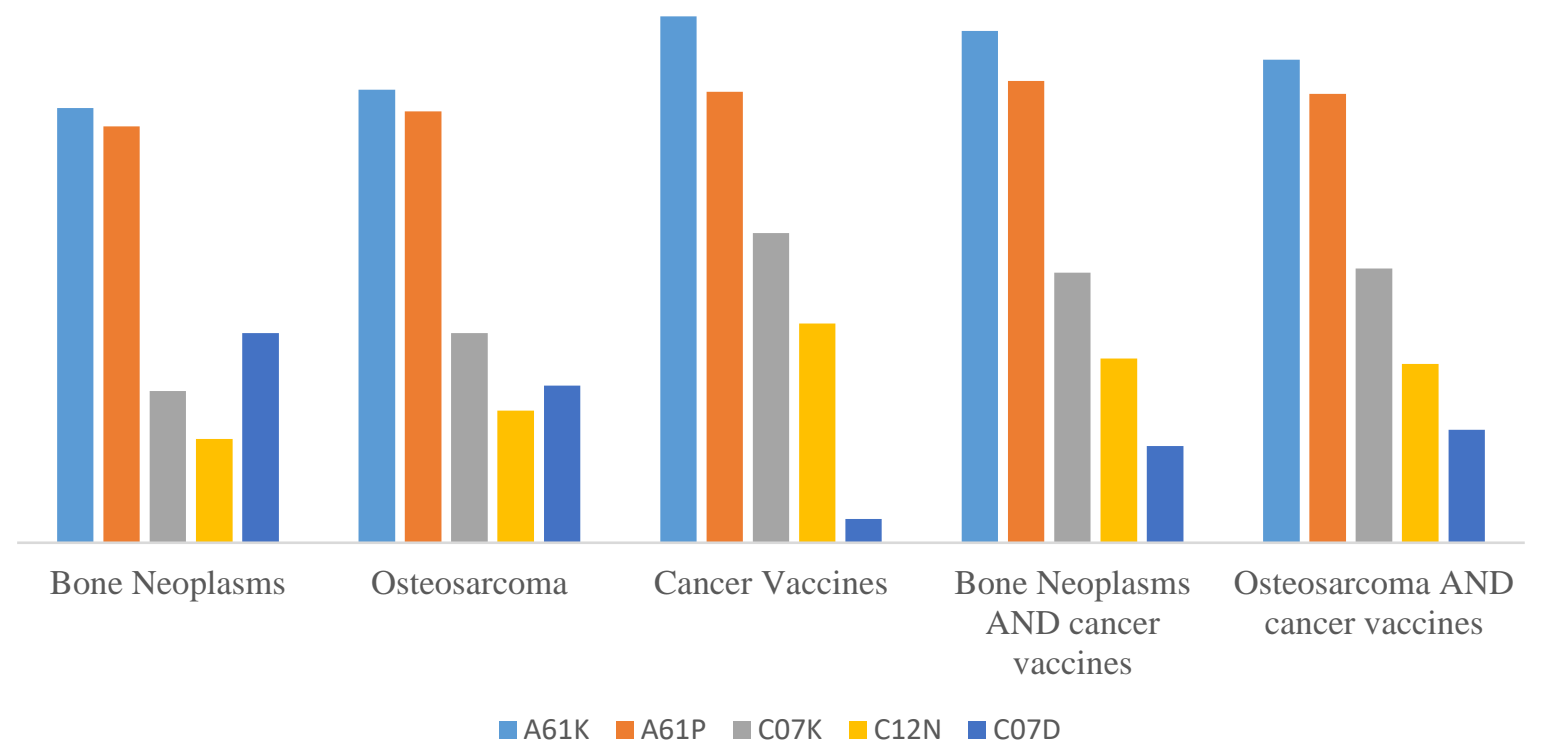

Fonte: Autores (2021).

Na Figura 4 é possível verificar os resultados obtidos em relação aos descritores e o escritório BR em português e seus respectivos códigos internacionais de patentes. Como esperado, baseado nos próprios dados obtidos neste estudo, o descritor que conseguiu permitir um maior direcionamento relacionado ao tema foi o "Osteossarcoma AND vacinas anticâncer" apresentando um total de 159 invenções.

Ao se analisar ambos escritórios, foi perceptível a prevalências dos mesmos códigos internacionais de patentes em todas as buscas com todas os descritores utilizados. O código A61K está relacionado a invenções contendo PREPARAÇÕES PARA FINALIDADES MÉDICAS, ODONTOLÓGICAS OU HIGIÊNICAS; Enquanto que o A61P envolve invenções cuja finalidade é ATIVIDADE TERAPÊUTICA ESPECÍFICA DE COMPOSTOS QUÍMICOS OU PREPARAÇÕES MEDICINAIS; Correlacionando as buscas com o C07K o qual envolve invenções cuja a finalidade é a utilização de PEPTÍDEOS; Correlacionando as buscas com o $\mathrm{C} 12 \mathrm{~N}$ o qual envolve invenções cuja finalidade é invenções contendo MICROORGANISMOS OU ENZIMAS; COMPOSIÇÕES DAS MESMAS; PROPAGANDO, PRESERVANDO OU MANTENDO MICRORGANISMOS; MUTAÇÃO OU ENGENHARIA GENÉTICA; MEIOS DE CULTURA; e por fim ao se correlacionar com o código C07D que engloba invenções com a finalidade em se utilizar COMPOSTOS HETEROCÍCLICOS. A compreensão e definição desses códigos servem para compreendermos quais tecnologias estão sendo mais utilizadas para esse tipo de terapêutica, nos fornecendo uma visão geral tanto nacional quanto internacional. 
Figura 4. Gráfico apresentando os códigos internacionais de patentes associados as invenções e seu descritores no escritório BR - Brasil. Dados obtidos pelo Google Patentes.

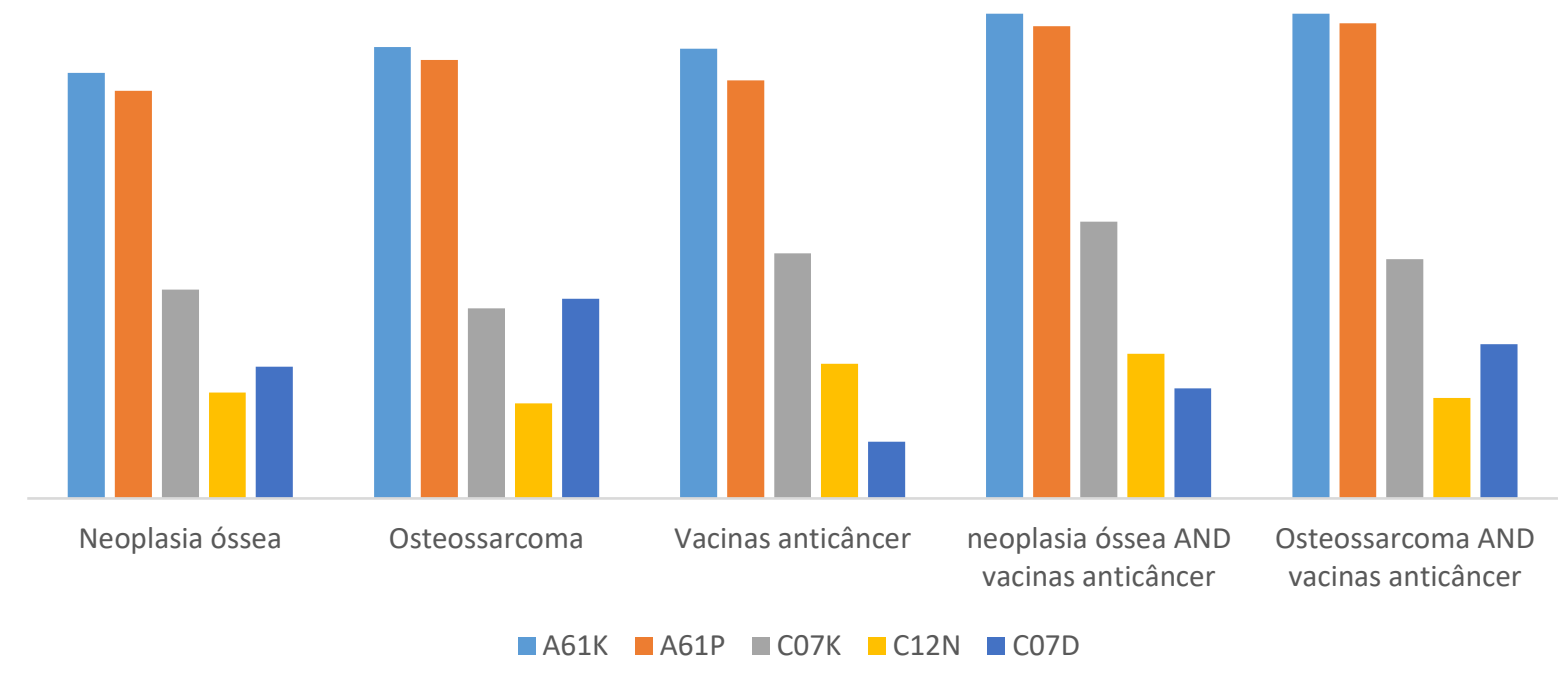

Fonte: Autores (2021).

Ao se analisar as patentes encontradas no escritório BR, devido ao quantitativo foi possível analisar criteriosamente essas invenções e algumas serão discutidas aqui. A invenção BR112013028887B1(BR112013028887B1 - Composição Imunogênica Apresentando Múltiplos Antígenos, e Métodos e Usos Dos Mesmos - Google Patents, n.d.) apresenta a utilização de diversos imunógenos que podem ser associados a diversas propostas vacinais como um adjuvante, mesma tecnologia é amplamente vista na literatura como uma opção viável. A invenção BR112015021414B1(BR112015021414B1 - Vírus Da Doença Newcastle e Seus Usos - Google Patents, n.d.) se refere a utilização do vírus quimérico da doença de Newcastle geneticamente modificado capaz de ativar os sinais coestimuladores ou inibi-los, podendo servir como uma plataforma também para diversos tipos de vacinas. Os inventores relatam que a invenção proporciona métodos de tratamento de câncer compreendendo administrar o vírus da doença de Newcastle em combinação com algum imunógeno de importância. Outras invenções propondo o uso de imunomoduladores também foram encontradas. A patente BR112013017096A2(BR112013017096A2 - Composição e Métodos de Indução de Resposta Citotóxica Dependente de Complemento Mediado Por Anticorpo Específico de Tumor Em Animal Tendo Tumor, de Geração de Anticorpos in Vivo, de Inibição Do Crescimento Ou Morte de Célula de Câncer, Para Tratar Indivíduo Com Câncer, Para Adaptar de Terapia de Câncer Para Indivíduo Com Câncer e Para Identificar Antígeno Específico de Tumor - Google Patents, n.d.) refere-se à utilização de vírus oncolíticos com a finalidade de ativar a resposta citotóxica dependente do sistema complemento. Os inventores relatam que a administração da composição proposta na patente possui a capacidade de induz no animal a produção de anticorpos que irão mediar uma resposta citotóxica específica para o referido tumor, podendo ser aplicado para diversos tumores, incluindo o osteossarcoma.

A invenção BR112020020085A2(BR112020020085A2 - Composto, Composição Farmacêutica, e, Métodos Para Induzir Uma Resposta Imune, Para Induzir Produção de Interferons de Tipo i Dependentes de Sting e Para Tratar Um Distúrbio de Proliferação Celular - Google Patents, n.d.) traz uma proposta em se utilizar uma composição farmacêutica capaz de ativar a resposta imune, para induzir a produção de interferons de tipo I dependentes de STING, e/ou para tratar um distúrbio de proliferação celular, como câncer, também fornece uma imunoterapia para diversos tipos de câncer e não apenas o osteossarcoma. A invenção BR112020012250A2(BR112020012250A2 - Terapia Anticâncer de Combinação Com Um Antagonista de Iap e 
Molécula Anti Pd-1 - Google Patents, n.d.) traz uma proposta diferente das demais, fornecendo um pré-tratamento utilizando um antagonista de IAP para aumentar a imunogenicidade do microambiente tumoral antes de um tratamento do indivíduo com uma molécula anti-PD-1. IAP são proteínas que apresentam em torno de três domínios de repetição de IAP baculoviral (BIR). Comumente as células humanas expressam em torno de 8 IAP e isso acaba inibindo a apoptose influenciando em diversos processos celulares, inclusive a regulação transcricional mediada pelo Fator Nuclear $\kappa B(N F-\kappa B)$ o qual é importante na expressão de genes que medeiam a inflamação, imunidade, sobrevivência celular, dentre outros. Essa regulação dependente dessas proteínas IAP geram um grande impacto no sistema imunológico e ao se falar sobre a imunidade tumoral esse impacto é ainda maior, visto que normalmente há falhas na imunidade tumoral. Ao se utilizar um antagonista dessas proteínas o microambiente terá capacidade em ativar a cascata imunológica, e ao se associar com um tratamento posterior com anti-PD-1 (anticorpo para o receptor PD-1). Já a invenção BR112019013282A2(BR112019013282A2 - Células Natural Killer Geneticamente Modificadas - Google Patents, n.d.) traz células natural killer modificadas geneticamente capazes de suprimir a proliferação de células tumorais ou para inibir a infecção por patógenos.

Ao se analisar as patentes encontradas no escritório BR foi possível verificar a importância da imunoterapia para alguns tipos de câncer, assim como para OS. Esses resultados sinalizam que pesquisas direcionadas para imunoterapias são crescentes e promissoras e podem fornecer melhor qualidade de vida para os pacientes com câncer. Outro ponto chave dessas buscas foi perceber ainda a carência de marcadores moleculares importantes de OS sendo utilizados como possíveis imunógenos vacinais, indicando talvez um caminho a seguir. O uso de vacinas terapêuticas é crescente e para diversos outros tipos de câncer já apresentam propostas em ensaios clínicos (Chevallier et al., 2021; del Giudice et al., 2021; Firnhaber et al., 2021; Ishikawa et al., 2021; Vreeland et al., 2021).

\section{Conclusão}

O OS é um dos principais cânceres ósseos e que afeta uma faixa etária muito diversa. O seu tratamento convencional acarreta uma diminuição na qualidade de vida do paciente os quais apresentam alta mortalidade. Diante disso, a busca recorrente por novos tratamentos ou até como adjuvantes dos tratamentos convencionais é crescente e ao se compreender a fisiopatologia do OS as imunoterapias surgem como uma estratégia importante que pode amenizar os efeitos adversos dos tratamentos convencionais.

Com isso esse trabalho buscou compreender o cenário relacionado a imunoterapia com enfoque em vacinas anticâncer, principalmente para OS. Utilizou-se três descritores obtidos no DeCs isoladamente ou associados e diante das buscas percebeuse que foi possível direcionar mais as buscas quando associou os descritores "Osteossarcoma AND vacinas anticâncer". Com esse descritor foi possível encontrar inúmeros artigos e invenções utilizando composições farmacêuticas como imunomoduladores, vírus oncolíticos como potenciais tratamentos de câncer, assim como vacinas de mRNA, vacinas com a fusão de células T $\gamma \delta$ alogênica e células tumorais e a utilização de nanopartículas carreando PD-1.

Foi possível perceber a diferença entre a prospecção científica e tecnológica, em que a prospecção científica apresentou propostas vacinais bem diferentes das patentes encontradas. Essa diferença é bem esperada diante do tema ser recente e as vacinas terapêuticas estarem sendo foco crescente em sua utilização para câncer nos últimos anos, possivelmente justificando a falta de tecnologias como está nas invenções já encontradas nos escritórios tanto do BR quanto WO. Com isso, vale ressaltar a importância de prospecções comparativas como está em que possibilita uma visão geral do cenário relacionado ao OS podendo direcionar as pesquisas futuras na tentativa de mitigar essa problemática.

Desse modo, sugere-se que pesquisas futuras direcionem estudos voltados as vacinas no tratamento do OS, bem como, tendo em vista que, atualmente, as vacinas terapêuticas são utilizadas por uma grande parcela da população, sobretudo, por apresentarem efeitos satisfatório, sendo aplicada a partir do tipo de patologia que o indivíduo está exposto, de modo a 
desmistificar que a ideia de vacinas terapêuticas com auxílio da bioinformática não são ofensivas e prejudiciais à saúde, ressaltando assim, a relevância do presente estudo.

\section{Referências}

Argenziano, M., Tortora, C., Pota, E., Paola, A. di, Martino, M. di, Leva, C. di, Pinto, D. di, \& Rossi, F. (2021). Osteosarcoma in Children: Not Only Chemotherapy. Pharmaceuticals 2021, Vol. 14, Page 923, 14(9), 923. https://doi.org/10.3390/PH14090923

Cabrera-Andrade, A., López-Cortés, A., Jaramillo-Koupermann, G., González-Díaz, H., Pazos, A., Munteanu, C. R., Pérez-Castillo, Y., \& Tejera, E. (2020). A Multi-Objective Approach for Anti-Osteosarcoma Cancer Agents Discovery through Drug Repurposing. Pharmaceuticals, 13(11), 1-16. https://doi.org/10.3390/PH13110409

Chevallier, P., Saiagh, S., Dehame, V., Guillaume, T., Peterlin, P., Bercegeay, S., le Bris, Y., Bossard, C., Gauvrit, I., Dreno, B., Juge-Morineau, N., Béné, M. C., \& Gregoire, M. (2021). A phase I/II feasibility vaccine study by autologous leukemic apoptotic corpse-pulsed dendritic cells for elderly AML patients. Human Vaccines \& Immunotherapeutics, 17(10), 3511-3514. https://doi.org/10.1080/21645515.2021.1943991

Corre, I., Verrecchia, F., Crenn, V., Redini, F., \& Trichet, V. (2020). The Osteosarcoma Microenvironment: A Complex but Targetable Ecosystem. Cells, 9(4). https://doi.org/10.3390/CELLS9040976

Cortini, M., Avnet, S., \& Baldini, N. (2017). Mesenchymal stroma: Role in osteosarcoma progression. Cancer Letters, 405, 90-99. https://doi.org/10.1016/J.CANLET.2017.07.024

Chueke, G. V., \& Amatucci, M. (2015). O que é bibliometria? Uma introdução ao Fórum. Internext, 10(2), 1-5. https://doi.org/10.18568/1980-4865.1021-5

del Giudice, F., Busetto, G. M., Gross, M. S., Maggi, M., Sciarra, A., Salciccia, S., Ferro, M., Sperduti, I., Flammia, S., Canale, V., Chung, B. I., Conti, S. L., Eisenberg, M. L., Skinner, E. C., \& de Berardinis, E. (2021). Efficacy of three BCG strains (Connaught, TICE and RIVM) with or without secondary resection (re-TUR) for intermediate/high-risk non-muscle-invasive bladder cancers: results from a retrospective single-institution cohort analysis. Journal of Cancer Research and Clinical Oncology, 147(10), 3073-3080. https://doi.org/10.1007/S00432-021-03571-0/FIGURES/2

Erp, A. E. M. van, Versleijen-Jonkers, Y. M. H., Hillebrandt-Roeffen, M. H. S., Houdt, L. van, Gorris, M. A. J., Dam, L. S. van, Mentzel, T., Weidema, M. E., Savci-Heijink, C. D., Desar, I. M. E., Merks, H. H. M., Noesel, M. M. van, Shipley, J., Graaf, W. T. A. van der, Flucke, U. E., Meyer-Wentrup, F. A. G., van Erp, A. E. M., Versleijen-Jonkers, Y. M. H., Hillebrandt-Roeffen, M. H. S., \& Meyer-Wentrup, F. A. G. (2017). Expression and clinical association of programmed cell death-1, programmed death-ligand-1 and CD8 + lymphocytes in primary sarcomas is subtype dependent. Oncotarget, 8(41), 71371-71384. https://doi.org/10.18632/ONCOTARGET.19071

F, J., S, J., S, S., A, N., A, S., D, R., \& S, D. (2020). Osteosarcoma: A comprehensive review of management and treatment strategies. Annals of Diagnostic Pathology, 49. https://doi.org/10.1016/J.ANNDIAGPATH.2020.151654

Firnhaber, C., Swarts, A., Jezile, V., Mulongo, M., Goeieman, B., Williams, S., Faesen, M., Michelow, P., \& Wilkin, T. (2021). Human Papillomavirus Vaccination Prior to Loop Electroexcision Procedure Does Not Prevent Recurrent Cervical High-grade Squamous Intraepithelial Lesions in Women Living With Human Immunodeficiency Virus: A Randomized, Double-blind, Placebo-controlled Trial. Clinical Infectious Diseases, 73(7), e2211-e2216. https://doi.org/10.1093/CID/CIAA1456

Guerra, M. R., Bustamante-Teixeira, M. T., Corrêa, C. S. L., de Abreu, D. M. X., Curado, M. P., Mooney, M., Naghavi, M., Teixeira, R., França, E. B., \& Malta, D. C. (2017). Magnitude e variação da carga da mortalidade por câncer no Brasil e Unidades da Federação, 1990 e 2015. Revista Brasileira de Epidemiologia, 20, 102-115. https://doi.org/10.1590/1980-5497201700050009

Hu, G., Cheng, Z., Wu, Z., \& Wang, H. (2019). Identification of potential key genes associated with osteosarcoma based on integrated bioinformatics analyses. Journal of Cellular Biochemistry, 120(8), 13554-13561. https://doi.org/10.1002/JCB.28630

IC, O., R, B., O, A., II, O., \& G, A. (2021). Evaluation of possible effects of Persea americana seeds on female reproductive hormonal and toxicity profile. Journal of Ethnopharmacology, 273. https://doi.org/10.1016/J.JEP.2021.113870

Ishikawa, T., Kageyama, S., Miyahara, Y., Okayama, T., Kokura, S., Wang, L., Sato, E., Yagita, H., Itoh, Y., \& Shiku, H. (2021). Safety and antibody immune response of CHP-NY-ESO-1 vaccine combined with poly-ICLC in advanced or recurrent esophageal cancer patients. Cancer Immunology, Immunotherapy 2021 70:11, 70(11), 3081-3091. https://doi.org/10.1007/S00262-021-02892-W

John D; WICK, M. R. (2018). The American Cancer Society's Principles of Oncology. The American Cancer Society's Principles of Oncology. https://doi.org/10.1002/9781119468868

Kaur Sarhadi, V., Daddali, R., Seppänen-Kaijansinkko, R., Pathogenesis, O., Klimczak, A., Kaltschmidt, C., \& Mertzlufft, F. (2021). Mesenchymal Stem Cells and Extracellular Vesicles in Osteosarcoma Pathogenesis and Therapy. International Journal of Molecular Sciences 2021, Vol. 22, Page 11035, 22(20), 11035. https://doi.org/10.3390/IJMS222011035

Lugowska, I., Teterycz, P., \& Rutkowski, P. (2018). Immunotherapy of melanoma. Contemporary Oncology (Poznan, Poland), 22(1A), 61-67. https://doi.org/10.5114/WO.2018.73889

M, P., Y, L., W, G., Z, S., Z, W., \& L, L. (2021). Recent Advances in Epigenetics of Macrovascular Complications in Diabetes Mellitus. Heart, Lung \& Circulation, 30(2), 186-196. https://doi.org/10.1016/J.HLC.2020.07.015

MA, A., C, E.-B., MH, E., YO, E., M, M., D, J., TS, A. S., F, K., C, M., E, M., I, O., N, D., \& AH, E. (2020). Novel therapeutic strategies for spinal osteosarcomas. Seminars in Cancer Biology, 64, 83-92. https://doi.org/10.1016/J.SEMCANCER.2019.05.018 
Marcião, L. G. de A., Sousa, V. N. F. de, Lima, P. E., Lima, L. H. C., Marcião, E. F., Alves, E. L. C., Lira, J. V. M., Moura, L. F. de, Olivetto, M. M. S., Silva, G. da, Ferreira, O. P. C., Rodrigues, A. G., Alves, C. P., Viana, J. B., \& Alencar, I. de. (2021). A importância da atenção Fisioterapêutica nos cuidados paliativos em pacientes com câncer. Research, Society and Development, 10(6), e46310616042. https://doi.org/10.33448/rsd-v10i6.16042

Martin, N. T., \& Bell, J. C. (2018). Oncolytic Virus Combination Therapy: Killing One Bird with Two Stones. Molecular Therapy, 26(6), 1414-1422. https://doi.org/10.1016/J.YMTHE.2018.04.001

MF, W., LM, W., \& TP, C. (2018). Immunotherapy for osteosarcoma: Where do we go from here? Pediatric Blood \& Cancer, 65(9). https://doi.org/10.1002/PBC.27227

Misaghi, A., Goldin, A., Awad, M., \& Kulidjian, A. A. (2018). Osteosarcoma: a comprehensive review. SICOT-J, 4. https://doi.org/10.1051/SICOTJ/2017028

Miwa, S., Shirai, T., Yamamoto, N., Hayashi, K., Takeuchi, A., Igarashi, K., \& Tsuchiya, H. (2019). Current and Emerging Targets in Immunotherapy for Osteosarcoma. Journal of Oncology, 2019. https://doi.org/10.1155/2019/7035045

Mutsaers, A. J., \& Walkley, C. R. (2014). Cells of origin in osteosarcoma: mesenchymal stem cells or osteoblast committed cells? Bone, 62, 56-63. https://doi.org/10.1016/J.BONE.2014.02.003

Saba, K. H., Cornmark, L., Rissler, M., Fioretos, T., Åström, K., Haglund, F., Rosenberg, A. E., Brosjö, O., \& Nord, K. H. (2019). Genetic profiling of a chondroblastoma-like osteosarcoma/malignant phosphaturic mesenchymal tumor of bone reveals a homozygous deletion of CDKN2A, intragenic deletion of DMD, and a targetable FN1-FGFR1 gene fusion. Genes, Chromosomes \& Cancer, 58(10), 731-736. https://doi.org/10.1002/GCC.22764

Sadykova, L. R., Ntekim, A. I., Muyangwa-Semenova, M., Rutland, C. S., Jeyapalan, J. N., Blatt, N., \& Rizvanov, A. A. (2020). Epidemiology and Risk Factors of Osteosarcoma. Cancer Investigation, 38(5), 259-269. https://doi.org/10.1080/07357907.2020.1768401

Tadano, H., Tsukahara, T., Mizushima, E., Akamatsu, A., Watanabe, K., Nojima, I., Kubo, T., Kanaseki, T., Hirohashi, Y., Sato, N., \& Torigoe, T. (2020). Development of an artificial antibody specific for HLA/peptide complex derived from cancer stem-like cell/cancer-initiating cell antigen DNAJB8. British Journal of Cancer 2020 123:9, 123(9), 1387-1394. https://doi.org/10.1038/s41416-020-1017-1

Verheust, C., Goossens, M., Pauwels, K., \& Breyer, D. (2012). Biosafety aspects of modified vaccinia virus Ankara (MVA)-based vectors used for gene therapy or vaccination. Vaccine, 30(16), 2623-2632. https://doi.org/10.1016/J.VACCINE.2012.02.016

Vreeland, T. J., Clifton, G. T., Hale, D. F., Chick, R. C., Hickerson, A. T., Cindass, J. L., Adams, A. M., Bohan, P. M. K., Andtbacka, R. H. I., Berger, A. C., Jakub, J. W., Sussman, J. J., Terando, A. M., Wagner, T., Peoples, G. E., \& Faries, M. B. (2021). A Phase IIb Randomized Controlled Trial of the TLPLDC Vaccine as Adjuvant Therapy After Surgical Resection of Stage III/IV Melanoma: A Primary Analysis. Annals of Surgical Oncology, $28(11)$, 6126-6137. https://doi.org/10.1245/S10434-021-09709-1/FIGURES/3

Wang, S. D., Li, H. Y., Li, B. H., Xie, T., Zhu, T., Sun, L. L., Ren, H. Y., \& Ye, Z. M. (2016). The role of CTLA-4 and PD-1 in anti-tumor immune response and their potential efficacy against osteosarcoma. International Immunopharmacology, 38, 81-89. https://doi.org/10.1016/J.INTIMP.2016.05.016

Watanabe, N., McKenna, M. K., Rosewell Shaw, A., \& Suzuki, M. (2021). Clinical CAR-T Cell and Oncolytic Virotherapy for Cancer Treatment. Molecular Therapy, 29(2), 505-520. https://doi.org/10.1016/J.YMTHE.2020.10.023

Y, Z., N, S., TT, C., O, K., RL, B., YB, M., HS, L., ES, K., \& SS, W. (2020). Vaccine efficacy against primary and metastatic cancer with in vitro-generated CD103 + conventional dendritic cells. Journal for Immunotherapy of Cancer, 8(1). https://doi.org/10.1136/JITC-2019-000474

Yalçın, S., Yalçınkaya, S., \& Ercan, F. (2021). In silico detection of inhibitor potential of Passiflora compounds against SARS-Cov-2(Covid-19) main protease by using molecular docking and dynamic analyses. Journal of Molecular Structure, 1240. https://doi.org/10.1016/J.MOLSTRUC.2021.130556

Younis, J., al Antably, I., Zamzam, M., Salem, H., Zaki, E., \& Hassanian, O. (2019). Role of 18 F-fluorodeoxyglucose positron emission tomography/computed tomography and magnetic resonance imaging in prediction of response to neoadjuvant chemotherapy in pediatric osteosarcoma. World Journal of Nuclear Medicine, 18(4), 378. https://doi.org/10.4103/WJNM.WJNM_52_18

Zhang, Y., Yang, J., Zhao, N., Wang, C., Kamar, S., Zhou, Y., He, Z., Yang, J., Sun, B., Shi, X., Han, L., \& Yang, Z. (2018). Progress in the chemotherapeutic treatment of osteosarcoma. Oncology Letters, 16(5), 6228. https://doi.org/10.3892/OL.2018.9434 